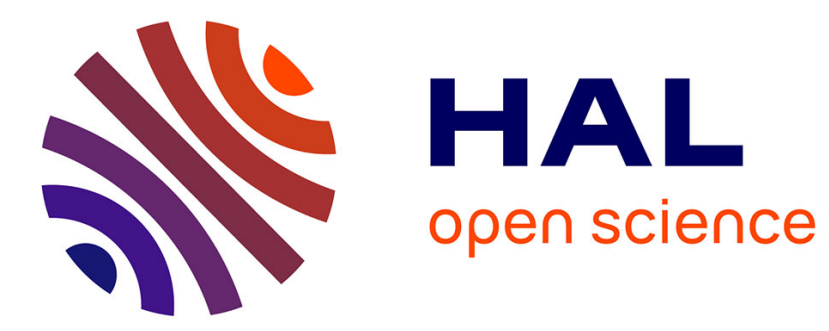

\title{
Thermal self-synchronization of nano-objects
}

Zhongwei Zhang, Yangyu Guo, Marc Bescond, Jie Chen, Masahiro Nomura, Sebastian Volz

\section{To cite this version:}

Zhongwei Zhang, Yangyu Guo, Marc Bescond, Jie Chen, Masahiro Nomura, et al.. Thermal self-synchronization of nano-objects. Journal of Applied Physics, 2021, 130 (8), pp.084301. $10.1063 / 5.0058252$. hal-03406404

\section{HAL Id: hal-03406404 https://hal.science/hal-03406404}

Submitted on 27 Oct 2021

HAL is a multi-disciplinary open access archive for the deposit and dissemination of scientific research documents, whether they are published or not. The documents may come from teaching and research institutions in France or abroad, or from public or private research centers.
L'archive ouverte pluridisciplinaire HAL, est destinée au dépôt et à la diffusion de documents scientifiques de niveau recherche, publiés ou non, émanant des établissements d'enseignement et de recherche français ou étrangers, des laboratoires publics ou privés. 


\title{
Thermal self-synchronization of nano-objects
}

\author{
Zhongwei Zhang, ${ }^{1,2,3}$ Yangyu Guo, ${ }^{3}$ Marc Bescond, ${ }^{4}$ Jie Chen, ${ }^{1,2, a)}$ Masahiro Nomura, ${ }^{3, \text { b) }}$ and Sebastian \\ Volz $2,4, c)$ \\ 1) Center for Phononics and Thermal Energy Science, School of Physics Science and Engineering, Tongji University, \\ 200092 Shanghai, PR China \\ ${ }^{2)}$ China-EU Joint Lab for Nanophononics, Tongji University, 200092 Shanghai, \\ PR China \\ ${ }^{3)}$ Institute of Industrial Science, The University of Tokyo, Tokyo 153-8505, Japan \\ ${ }^{4)}$ Laboratory for Integrated Micro and Mechatronic Systems, CNRS-IIS UMI 2820, The University of Tokyo, \\ Tokyo 153-8505, Japan
}

(Dated: 4 July 2021)

Self-synchronization is a ubiquitous phenomenon in nature, in which oscillators are collectively locked in frequency and phase through mutual interactions. While self-synchronization requires the forced excitation of at least one of the oscillators, we demonstrate that this mechanism spontaneously appears due to the activation from thermal fluctuations. By performing molecular dynamics simulations, we demonstrate selfsynchronization in a platform supporting doped silicon resonator nanopillars having different eigenfrequencies. We find that pillar's vibrations are spontaneously converging to the same frequency and phase. In addition, the dependencies on intrinsic frequency difference and coupling strength agree well with the Kuramoto model predictions. More interestingly, we find that a balance between energy dissipation resulting from phononphonon scattering and potential energy between oscillators is reached to maintain synchronization. The balance could be suppressed by increasing the membrane size. While microscopic stochastic motions are known to follow random probability distributions, we finally prove that they also can yield coherent collective motions via self-synchronization.

\section{INTRODUCTION}

Self-synchronization of a population of coupled oscillators is a common phenomenon in nature, as observed in a wide range of physical and biological systems $^{1-8}$. Through mutual interactions, oscillators are self-organized into a collective motion, in which all synchronized units are locked to a single frequency and phase $^{1,4,9,10}$. This mechanism was pioneered by $\mathrm{Ku}-$ ramoto, and his model has been serving as a reference in numerous situations ${ }^{1,9,11}$. Self-synchronization in many fields has attracted continuous attention, for example to achieve coherent operation of micromechanical oscillators in optomechanics ${ }^{4,6,12-14}$ and to understand the transient cellular differentiation in biological systems ${ }^{2,15}$.

Usually, synchronization is understood as a stationary state sustained by external forces ${ }^{1,8,16}$. Studies $^{16-18}$ demonstrated that the rate of change of the entropy $S$ for the system can be properly decomposed as

$$
\frac{d S}{d t}=\Pi-\Phi
$$

where $\Pi$ is the entropy production and $\Phi$ refers to the entropy flux. Specifically, $\Pi$ is related to the irreversible thermal dissipation inside the system, while $\Phi$ is always arising from the external drivings. The stationary state

\footnotetext{
a)Electronic mail: jie@tongji.edu.cn

b) Electronic mail: nomura@iis.u-tokyo.ac.jp

c) Electronic mail: volz@iis.u-tokyo.ac.jp
}

of synchronization corresponds to the condition of $\Pi=\Phi$, indicating that the entropy rate $d S / d t=0^{7,8,16,19}$. For instance, Zhang et al. ${ }^{8}$ demonstrated that the stationary state of synchronization breaks the detailed balance by dissipation (i.e. related to $\Pi$ in Eq. (1)) and thus a continuous energy input (i.e. contributed by $\Phi$ in Eq. (1)) is needed to maintain it (i.e. $\Pi=\Phi$ ). Moreover, in a coupled optomechanical system, Lipson's group ${ }^{6}$ found that self-synchronization of micromechanical oscillators can be controlled by external optical driving action. Sheng et $a l .{ }^{5}$ also observed the phase locking between self-organized synchronous optomechanical oscillators in phonon lasers when the input driving power reaches the required level. On the other hand, the works from Bonilla et al. ${ }^{3}$, Zhang et al. ${ }^{8}$, and Blekhman et al. ${ }^{19}$ showed that the driving action for self-synchronization can also originate from within the system. As demonstrated by Zhang et $a l .^{8}$, the energy cost for the synchronization of coupled molecular oscillators in an isolated system can be provided by the hydrolysis of the molecule. In other words, the stationary state of selfsynchronization can be spontaneously achieved without external driving action. This self-synchronization is believed to be key to spontaneous formation of rhythms in nature as well as in statistical physics ${ }^{1,20,21}$.

Using direct simulation, we will consider an isolated system of coupled nano-objects where the sole source of vibrations can only be thermal fluctuations. These random fluctuations are expected to excite the eigenmodes of the nano-objects. However, under certain coupling conditions, self-synchronization should drive the random individual motions towards a collective uniform one. We finally aim at proving the existence of this particular 


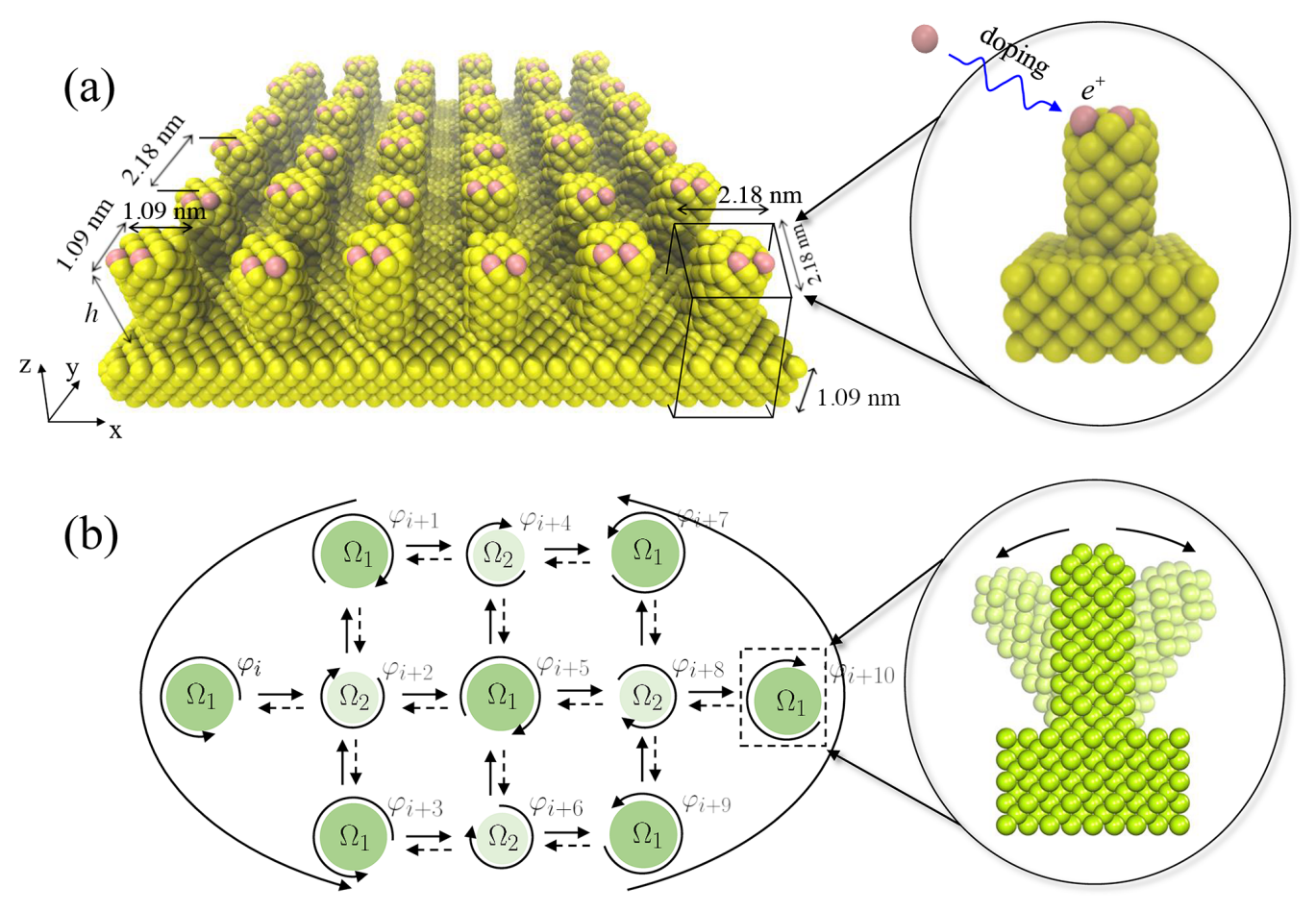

FIG. 1. Schematic figure of the doped silicon resonator system. (a) Pillared silicon membrane with electron doping (pink atoms) on the top of pillars. Pillars behave as resonators activated by thermal phonons. The zoom-in figure shows a unit of resonator. The dimensions of pillars and membrane are pointed and $h$ refers to the height of the pillars. (b) Schematic figure of the frequencies $(\Omega)$ and phase $(\varphi)$ dynamics of resonators under mean-field coupling. The system consists of two type resonators with frequencies $\Omega_{1}$ and $\Omega_{2}$, and random phases $\varphi_{i, i+1, \ldots}$. The long arrows indicate the collective motion of all resonators in a synchronized system. The zoom-in figure shows the vibration of a pillar as a unit of the resonator.

state of synchronization where acoustic (coherent) and thermal (incoherent) vibrations (or phonons) cooperate. While microscopic stochastic motions are known to follow random probability distributions, we will prove that they can also spontaneously yield coherent collective behaviours. As demonstrated before, the coherence of thermal phonons plays a crucial impact on the thermal transport in bulk materials and phononic crystals ${ }^{22-28}$. The self-synchronization is also expected to yield understanding and guidelines for the engineering of the coherence for thermal phonons.

In the following, the terms synchronization and selfsynchronization will be used indifferently to denominate the onset of a coherent motion of nano-objects without any external driving action. In contrast to usual selfsynchronized systems, we highlight the absence of external driving action here. Synchronizations of frequency and phase are proven and investigated. The relevance of previous theoretical models is confirmed in the present frame of thermal phonon activated resonators. The effects of frequency difference, coupling strength, and temperature on synchronization are discussed. In addition, the generation of coherent vibrations after synchronization is also investigated through a wavelet transform approach. Proving self-synchronization establishes another framework for the understanding of the dynamics of cou- pled resonators and provides a new route for the generation of coherent thermal vibrations (phonons).

\section{METHODOLOGY}

To directly investigate the dynamics of coupled oscillators, we consider a silicon pillared membrane as shown in Fig. 1(a). In a unit, the dimensions of the membrane element are fixed to $2.18 \mathrm{~nm} \times 2.18 \mathrm{~nm} \times 1.09 \mathrm{~nm}$, while the dimensions of the pillars are $1.09 \mathrm{~nm} \times 1.09 \mathrm{~nm}$ in $\mathrm{x}-\mathrm{y}$ directions. Thus, the distance between neighbor pillars (center to center) is set to $2.18 \mathrm{~nm}$. The dimensions of pillars and membrane are shown in Fig. 1(a). Previous studies ${ }^{29,30}$ found that pillars on the surface of a membrane act as local resonators and their predominant resonance frequency is expectedly decreasing with the pillar height ${ }^{31,32}$. To achieve frequency difference between the resonators, we thus consider two types of pillars with different heights, i.e. $h_{1}$ and $h_{2}$, with corresponding frequencies $\Omega_{1}$ and $\Omega_{2}$. That is, we model two types of units with different pillar heights on the membrane. By setting a $16 \times 16$ supercell of intercalated units, a resonator system containing two intrinsic frequencies is obtained, while phases $\varphi$ of pillars are initially randomized via MD simulation (See Fig. 1(b)). Here, the intercalated lay- 
out corresponds to a distribution of two types of pillars along $\mathrm{x}$ direction as $12121212 \ldots$, where 1 refers to pillar1 and 2 to pillar-2. Moreover, two frequency differences, $\delta \Omega=\left|\Omega_{1}-\Omega_{2}\right|$, are studied by varying the pillar height, $\delta \Omega_{I}=0.02 \mathrm{THz}$ (with $h_{1}=2.4 \mathrm{~nm}$ and $h_{2}=3.3 \mathrm{~nm}$ ) and $\delta \Omega_{I I}=0.04 \mathrm{THz}$ (with $h_{1}=2.4 \mathrm{~nm}$ and $h_{2}=4.2 \mathrm{~nm}$ ). The resonance frequencies of the pillars are obtained from the vibrational density of states calculations as discussed in Sec. IV.

However, the relatively long distance between pillars yields negligible covalent bondings. To introduce a meanfield coupling between resonators, a long-range electrostatic force is added by doping the top of the pillars, as shown in Fig. 1(a). In simulations, the doping is modeled by adding electrons to atoms and the doping ratio is defined as $n_{e} / n_{\text {atom }} \times 100 \%$. Here, $n_{e}$ is the number of electrons induced per pillar and $n_{\text {atom }}$ is the number of atoms per pillar. By varying the doping ratio, four systems are investigated, i.e. $\delta \Omega_{I}$ with doping ratio $0.2 \%$ (system I1) and $0.4 \%$ (system I2), and $\delta \Omega_{I I}$ with doping ratio $0.2 \%$ (system III) and $0.4 \%$ (system II2). Li et $a l .{ }^{33}$ found that the dopants in a confined nanostructure can be treated as localized charges, which corresponds to our doping model.

We use classical MD simulations to study the collective dynamics of nano-resonators. The covalent Si-Si interaction is modelled by the Tersoff potential ${ }^{34}$. The electrostatic interaction between dopants is modeled by the standard coulombic formula with a cutoff distance of $25.0 \AA$ and longer-range interactions are simulated by the $p p p m$ kspace method ${ }^{35}$. Periodic boundary conditions are applied in the $\mathrm{x}$ and $\mathrm{y}$ directions. Compared to the strong covalent bonding, the long-range electrostatic interaction is several orders of magnitude smaller, which means that the intrinsic frequencies of the resonators are not altered by the electrostatic forces (See Appendix A). All MD simulations are performed by using the LAMMPS package ${ }^{36}$ with a timestep of 0.35 fs. Firstly, the system is relaxed in the isothermal-isobaric (NPT) ensemble with $10^{5}$ steps. Then, the simulation runs over $2 \times 10^{5}$ steps with constant temperature in the canonical (NVT) ensemble. During these two processes, the dynamics of thermal phonons is controlled by the Nosé-Hoover thermostat ${ }^{37}$. Then, the simulation is carried out within the microcanonical (NVE) ensemble. Note that the choice of thermostat has a neglectable effect on the synchronization process (See Appendix B).

During the MD simulations, the pillared membranes show high stability with limited surface reconstruction as previously studied ${ }^{31,32,38}$. Considering that coherence ${ }^{24}$ and synchronization (See Sec. V) can be suppressed by the enhanced phonon-phonon scattering, we chose a relatively low temperature of $100 \mathrm{~K}$ in the study of synchronization process. The temperature effect on synchronization is also discussed in the Sec. V.

\section{THEORY}

Previously, synchronization dynamics of oscillators in optomechanics and other classical systems have been well understood by using theoretical models, in particular the Kuramoto model ${ }^{1,8,9,12,13}$. The Kuramoto model provides a fundamental description of self-synchronization of coupled resonators. It describes a non-linearly coupled system of $N$ oscillators with phases $\varphi_{i}$ and intrinsic frequency $\Omega_{i}$. For a two resonators system, the dynamics of the phase difference $\delta \varphi$ is described according to ${ }^{9}$

$$
\delta \dot{\varphi}=\delta \Omega-2 K \sin (\delta \varphi),
$$

where $\delta \Omega \equiv \Delta \Omega$ is the difference between two eigenfrequencies, and $K$ is the reduced coupling constant between resonators. Here, $K$ is normalized by the mass $M$ and the resonance frequency $\Omega$ of each pillar ${ }^{12}$ as follows

$$
K=\frac{\phi}{M \Omega},
$$

where $\phi$ refers to the harmonic force constant between oscillators. In this work, $\phi$ is obtained by fitting the potential energy surface between oscillators ${ }^{39}$ which is essentially generated by the electrostatic force. At frequency synchronization, one should find $\delta \dot{\varphi}=0$ and $K=\frac{\delta \Omega}{2 \sin (\delta \varphi)}$. Because $1 / \sin (\delta \varphi) \geq 1$, a threshold of the coupling constant $\left(K_{c}\right)$ for frequency synchronization can be defined by $1 / \sin (\delta \varphi)=1$. Therefore,

$$
K_{c}=\frac{\delta \Omega}{2} .
$$

When the coupling constant $K$ exceeds the threshold $K_{c}$, frequency synchronization is appearing ${ }^{1,9}$. Moreover, the phase synchronization happens when the phase lag $\delta \varphi \rightarrow 0$, i.e. $K>K_{c}$, indicating that the coupling constant $K$ for phase synchronization should be larger than the threshold. Accordingly, the condition for phase synchronization appears to be stricter than the one for frequency synchronization, which has been justified before for optomechanical systems ${ }^{1,4,12}$. Some revisions to Kuramoto model are further proposed ${ }^{12,40}$ as the dynamics of frequency and phase become more complex. The Kuramoto model is widely verified in mechanical ${ }^{1,12,13}$ and biological ${ }^{7,8}$ systems, its applicability to systems solely activated by thermal fluctuations remains to be discussed.

\section{RESULTS}

\section{A. Self-synchronization in frequency}

The frequency information of the resonators is obtained from the vibrational density of states $(v D O S)$, 


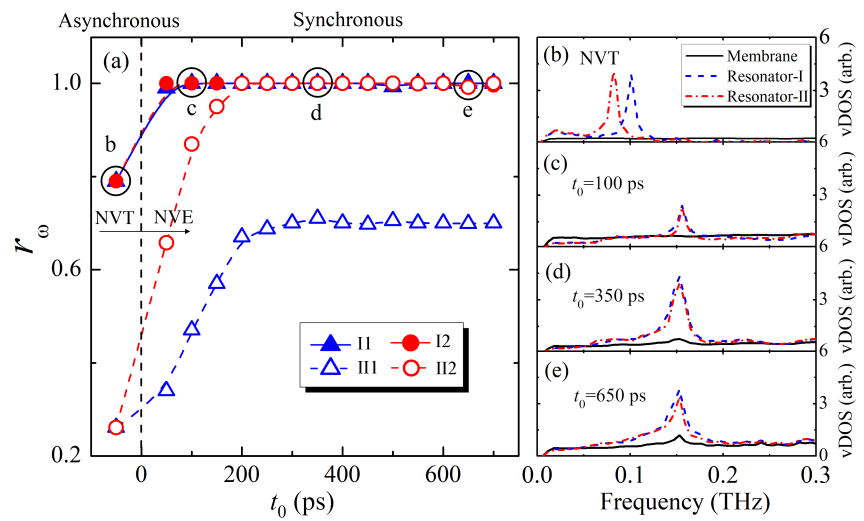

FIG. 2. (a) Synchronization degree in frequency $\left(r_{\omega}\right)$ versus evolution time for four systems, i.e. I1, I2, II1 and II2. (b-e) Vibrational density of states $(v D O S)$ of membrane, resonator-I and -II for the I1 system at different times. Times are also referenced as black circles in Fig. 2(a). The calculations are carried out at $100 \mathrm{~K}$.

$$
v \operatorname{DOS}(\omega)=\frac{1}{n_{a}} \sum_{i, \alpha}\left|\int_{t_{1}}^{t_{2}} v_{i, \alpha}(t) e^{i \omega t} d t\right|^{2} .
$$

$v_{i, \alpha}(t)$ refers to the atomic velocity of the $i$-th atom along the $\alpha$ direction at time $t$, and $n_{a}$ is the number of atoms in the summation. The integration corresponds to the time interval from $t_{1}$ to $t_{2}$ over which the spectral information is averaged. According to our further calculations in Appendix C, the vDOS from atomic displacements can also provide the same information on resnonance for pillars. Furthermore, as averaging over the atoms inside each pillar, as implemented in the phase analysis in the next subsection, the same resonant frequency can still be identified due to the predominance of resonant vibrations in pillars.

All the system atoms are involved in the sum and the obtained $v D O S$ will be associated to the time $t_{0}=\left(t_{1}+t_{2}\right) / 2$. $t_{1}$ and $t_{2}$ are defined as the limits of the time interval $50 \mathrm{ps}$ in duration and centered on the time variable $t_{0}$. In our simulations, $t_{0}=0$ refers to the time switching from NVT to NVE ensembles. The calculated $v D O S$ is shown in Figs. 2(b-e), in which the peaks correspond to the resonance frequencies of different types of pillars. The intrinsic frequency difference $(\delta \Omega)$ in Sec. II is also obtained from the $v D O S$ spectrum but in the undoped systems. Then, the synchronization degree in frequency can be defined as

$$
r_{\omega}=1-\frac{\Delta \Omega}{\bar{\Omega}},
$$

where, $\Delta \Omega$ is the frequency difference defined by subtracting the peak positions of the time-dependent $v D O S$ in Fig. 2(b-e) and $\bar{\Omega}$ denotes the averaged frequency. $r_{\omega}=1$ means that the system is fully synchronized in frequency, while in the asynchronous or partially synchronous state, $r_{\omega}$ remains always smaller than unity (See Fig. 2(a)).

The calculated $v D O S$ in the NVT ensemble in Fig. 2 (b) reveals that the pillars have different resonance frequencies, indicating the asynchronous state when $r_{\omega}<1$ (See Fig. 2(a)). Due to the weak long-range electrostatic force and the asynchronous dynamics between pillars, our calculations further indicate that the pillars in the NVT ensemble have the same resonance frequencies as the ones of the undoped systems. When switching from NVT to NVE ensembles $\left(t_{0} \geq 0\right)$, the dynamics of the coupled resonators becomes unconstrained. The selfsynchronization in frequency rapidly emerges. As shown in Fig. 2(a), synchronization degree $r_{\omega}$ is increasing with the evolution time. For the I1 system, the pillars are quickly synchronized to the same frequency $\left(\omega_{s}\right)$, which is manifested by the degenerated peaks in the $v D O S$ spectrum (See Figs. 2(c-e)). Because of the negligible effect of electrostatic interactions on the intrinsic vibration properties (See Appendix A), we can conclude that the observed frequency change and the degeneration of vibrational properties for different resonators are originating from the effect of self-synchronization activated by thermal fluctuations. Note that the synchronization of pillars also introduces the vibration of the membrane at the synchronization frequency $\omega_{s}$, as revealed by the increased amplitude of the membrane $v D O S$ in Fig. 2(e).

Moreover, the effect of intrinsic frequency difference and of coupling strength between resonators on the frequency self-synchronization is revealing consistency with theoretical predictions. The Kuramoto model in Eq. (2) indicates that self-synchronization in frequency can be enhanced by decreasing frequency difference or increasing the coupling strength. Correspondingly, the simulation results in Fig. 2(a) show that the system with small frequency difference $\left(\delta \Omega_{I}\right)$ or with high doping ratio $(0.4$ $\%)$ are more easily synchronized.

We also quantitatively explain the comparison between Eq. (2) and results in Fig. 2(a). The threshold of the coupling constant $\left(K_{c}\right)$ in Eq. (3) can correspond to a threshold criterium for the harmonic force constant $\left(\phi_{c}\right)$ in our pillar systems from Eq. (4). $\phi_{c}$ can be defined as

$$
\phi_{c}=\frac{M \delta \Omega \bar{\Omega}}{2},
$$

where $\bar{\Omega}$ is the averaged intrinsic frequency, i.e. $\bar{\Omega}=$ $\left(\Omega_{1}+\Omega_{2}\right) / 2$. For the $\delta \Omega_{1}$ and $\delta \Omega_{2}$ systems, we respectively obtain the threshold $\phi_{c}$ as $3.67 \times 10^{-3} \mathrm{eV} / \AA^{2}$ and $7.34 \times 10^{-3} \mathrm{eV} / \AA^{2}$. As the coupling constant exceeds the threshold value, the resonators begin to synchronize. The real coupling constant between pillars are calculated as $1.32 \times 10^{-2} \mathrm{eV} / \AA^{2}$ for the $0.2 \%$ doped systems and $8.79 \times 10^{-2} \mathrm{eV} / \AA^{2}$ for the $0.4 \%$ doped systems. Therefore, as we observed in Fig. 2(a), the systems with $0.4 \%$ doping are fully synchronized due to significantly larger 
coupling constants than the threshold one, while the $\delta \Omega_{2}$ systems with $0.2 \%$ doping, i.e. the II1 system, is partially synchronized because of the relatively larger threshold and lower coupling constant between pillars. The quantitative discussion above indicates relevant predictions from the Kuramoto model.

In addition, we find that the I1, I2 and II2 systems are fully synchronized at the frequencies $\omega_{s}$ of $0.152 \mathrm{THz}$, $0.167 \mathrm{THz}$ and $0.195 \mathrm{THz}$. In other words, the synchronization frequency is correlated with the system parameters: a larger averaged frequency (from the I2 to the II2 system) or a higher coupling constant between resonators (from the I1 to the I2 system) have a higher synchronization frequency.

\section{B. Self-synchronization in phase}

On the other hand, resonators can also be synchronized in phase. The synchronization degree in phase $\left(r_{p}\right)$ reads $^{1}$

$$
r_{p} e^{i \Theta}=\frac{1}{N} \sum_{i} e^{i \varphi_{i}}
$$

where $\Theta$ denotes the phase average. The $e^{i \varphi_{i}}$ term is calculated as the normalized displacement of the $i$-th resonator in the MD simulations. The displacement is the difference between the pillar position and the equilibrium one, i.e. $\mathbf{x}_{i}(t)=\left\langle\mathbf{x}_{i j}(t)\right\rangle_{j}-\left\langle\mathbf{x}_{i j}(t)\right\rangle_{j, t}$. Here, $\mathbf{x}_{i j}(t)$ is the position of the $j$-th atom in the $i$-th pillar at time $t$. That is, $\left\langle\mathbf{x}_{i j}(t)\right\rangle_{j}$ is the time dependent position of the $i$-th pillar as averaged over the atoms $(j)$ in each pillar, and $\left\langle\mathbf{x}_{i j}(t)\right\rangle_{j, t}$ is the mean position of the $i$-th pillar as averaged over the atoms $(j)$ and the simulation time $(t)$. Then, the displacement is normalized by the time averaged amplitude of the displacement. Before synchro-

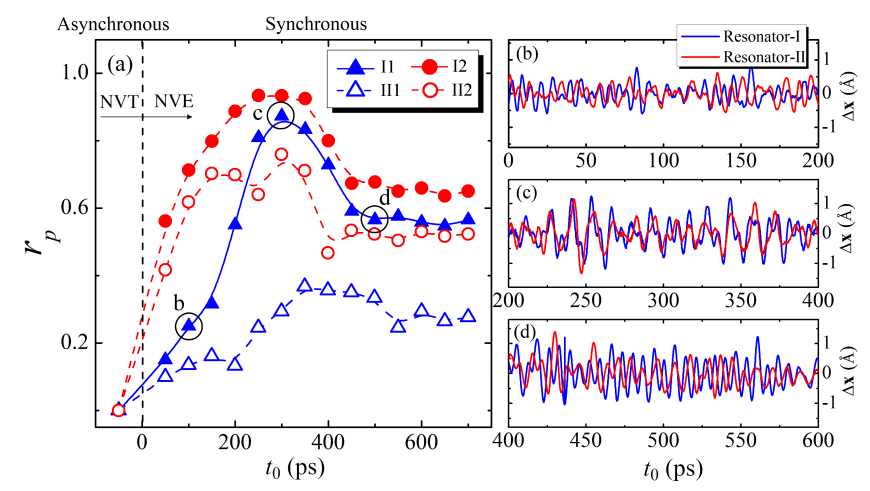

FIG. 3. (a) Synchronization degree in phase $\left(r_{p}\right)$ versus evolution time for four systems, i.e. I1, I2, II1 and II2. (b-d) Averaged displacement of two neighbouring resonators versus evolution time for the I1 system. Times are also referred to as black circles in Figs. 3(a). The calculations are carried out at $100 \mathrm{~K}$. nization, the resonators in the NVT ensemble exhibit uncorrelated dynamics (See Fig. 3(b)), as $r_{p} \approx 0.0$.

Under the free condition of the NVE ensemble, the transient process of phase synchronization is investigated in Fig. 3. Compared to the rapid and monotonous evolution of the frequency synchronization, the resonators are gradually phase synchronized and synchronization degree reaches its highest point around 300 ps with $r_{p} \approx 0.9$ in the case of I2 system. The displacement dynamics of the two types of resonators agree well (See Fig. 3(c)), exhibiting an excellent collective synchronized state. However, after 300 ps $r_{p}$ is decreasing with time and in the last stage $r_{p}$ remains at a stationary state after $500 \mathrm{ps,}$ indicating an only partial phase synchronization (Fig. $3(\mathrm{~d}))$.

We find that the phase synchronization of the pillar resonators also depends on the frequency difference between resonators and on the coupling strength due to doping in a similar way than for frequency synchronization. If we reduce the frequency difference and improve coupling strength, the phase synchronization can be enhanced. Moreover, compared to the synchronization in frequency, the phase synchronization is more difficult to achieve, especially the fully synchronized state, which agrees well with the prediction of the Kuramoto model as discussed in Sec. III. Obviously, the phase synchronization process requires a deeper insight more specifically regarding its dynamics.

\section{v. DISCUSSION}

\section{A. Entropy change}

When phases synchronize, even partially, resonators vibrate in an ordered or coherent state. Thus, selfsynchronization transition, including partial phase synchronization as we discussed in the above section, can spontaneously lead resonators from a disordered state to an ordered one. Apparently, this transition to a coherent motion is related to the variation of the state number and also of the entropy. To provide an insight in the detailed mechanisms occurring during self-synchronization, the entropy is calculated from the MD simulations ${ }^{41,42}$ as

$$
S=\frac{k_{B}}{2} \ln \operatorname{det}\left(\frac{k_{B} T e^{2}}{\hbar^{2}} \mathbf{M} \sigma+\mathbf{1}\right)
$$

where $k_{B}$ refers to the Boltzmann constant, $\hbar$ is the reduced Planck constant, $e$ is the Euler's number and $T$ corresponds to the temperature. $\mathbf{M}$ and $\mathbf{1}$ are the mass matrix and the unity matrix, respectively. $\sigma$ is the covariance matrix of the coordinate fluctuations, with $\sigma_{i j}=\left\langle\left(x_{i}-\left\langle x_{i}\right\rangle\right)\left(x_{j}-\left\langle x_{j}\right\rangle\right)\right\rangle$. The calculated entropy for the whole system and the resonator part for the I1 system are shown in Fig. 4(a). Before 300 ps, the entropy 

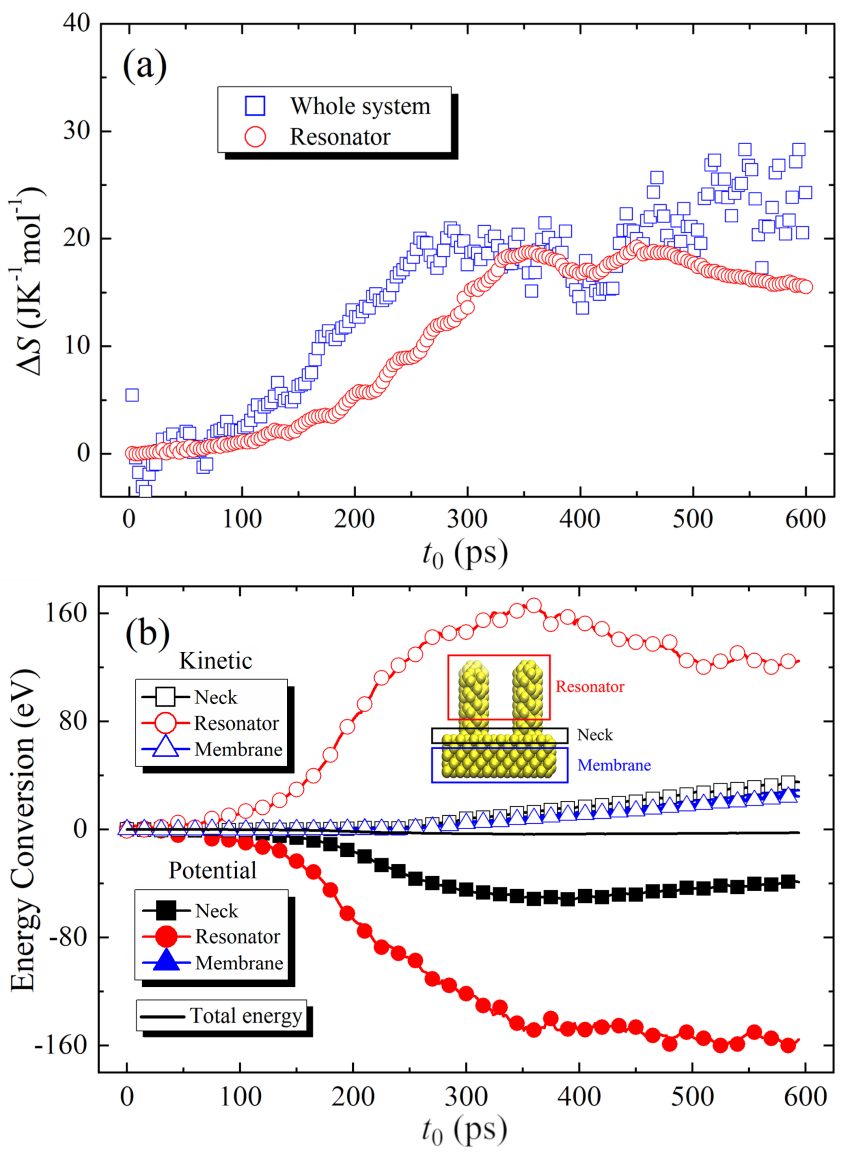

FIG. 4. Entropy change and energy conversion during selfsynchronization. (a) Entropy change of the whole system and of the resonators versus evolution time. (b) Kinetic energy and potential energy of neck, resonator and membrane regions and total energy versus evolution time of self-synchronization. The inset figure shows the definition of the neck, resonator and membrane regions. The I1 system is studied and its temperature is set to $100 \mathrm{~K}$ in both (a) and (b).

is continuously increasing, which corresponds to the synchronization process in Fig. 3(a) and resonators contribution predominates the entropy change. Therefore, the process of self-synchronization activated by thermal vibrations indeed follows the second law of thermodynamics.

After $300 \mathrm{ps}$, the entropy of the resonators and the whole system reaches a stationary-like state with small fluctuations, but still in agreement with the second law of thermodynamics. Compared to the one of the resonator, the intensive fluctuation of the entropy change of whole system should result from the larger number of atoms and also the fluctuation of energy at synchronization state in the membrane. From Eq. (1), we know that the entropy change due to the irreversible processes inside the system (П) should be equal to a driving power $(\Phi)$ that can only emanate from within the system itself. It should be noted that for the externally driven systems, $\Phi$ in Eq. (1) is the entropy flow from the exact external driving. It can however not be explicitly defined in our system, due to the fact that the driving from thermal fluctuations $\Phi$ occurs inside the system. Therefore, the use of $\Phi$ is an analog to the preconditions for synchronization in the previous studies ${ }^{16-18}$.

\section{B. Energy conversion}

To further investigate the transformation during selfsynchronization, we analyze the energy conversion in different regions of the system. Considering the possible strain in the neck region between pillars and membrane, we divide the system into three regions, i.e. neck, resonator and membrane, as shown in the inset figure of Fig. 4(b). The potential energy and kinetic energy is calculated respectively by summing atomic energies.

Fig. 4(b) shows that there is an obvious energy conversion between potential and kinetic energies, especially in the pillars. At initial state $\left(t_{0}=0\right)$, pillars vibrate in an asynchronous beat, in which the large relative displacement between them results in high amplitudes in the time-dependent potential energy. Before 300 ps, the relative displacement between pillars is spontaneously decreasing to minimize potential energy (See Fig. 4(b)). As the relative displacement reduces, pillars are vibrating in phase $^{43}$ (See the Video in Supplementary Material) and potential energy inside resonators and neck is converted into kinetic energy. As highlighted in Eq. 9, entropy, in turn, is simultaneously increasing with kinetic energy and temperature. The variation of the relative displacement between pillars with synchronization is further analyzed in the subsequent discussion.

The energy transfer between pillars and membrane slightly increases both potential and kinetic energies in the membrane (See Fig. 4(b)). Our further longer timescale simulation in Appendix D shows that the energies in the membrane would be rapidly converged and have high stability, which is well consistent with the stable synchronization state in Figs. 2 and 3. Moreover, the membrane size effect on the above energy conversion is also discussed in Appendix D. The increase in membrane size suppresses the conversion between potential and kinetic energies inside the pillars, which might result in the weakened synchronization degree. Remarkably, our additional simulation demonstrates that the membrane motion appears as a necessary mechanism to achieve synchronization, as the absence of synchronization was indeed observed when the in-plane degrees of freedom were removed in the membrane, which corresponds to the much larger or infinite membrane. However, because of the constrained connection between pillars and membrane, i.e. the neck part, the energies of the membrane shows weak dependence on the membrane thickness (See Appendix D), indicating the membrane does not directly involve the energy conversion.

Further analysis should be implemented to understand the partial desynchronization after 300 ps. Consider- 


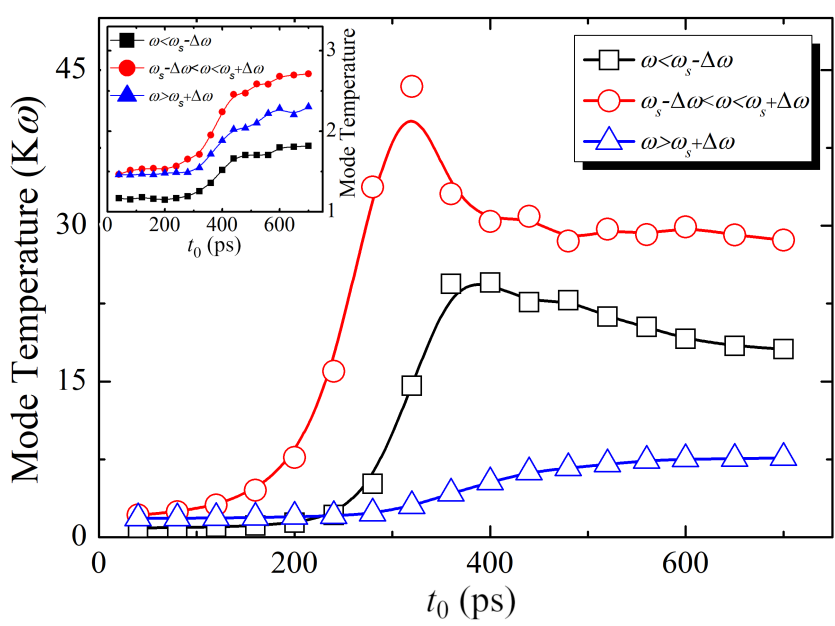

FIG. 5. Mode temperature evolution during selfsynchronization of resonators of frequency regions I) $\omega<$ $\omega_{s}-\Delta \omega$, II) $\omega_{s}-\Delta \omega<\omega<\omega_{s}+\Delta \omega$ and III) $\omega_{s}+\Delta \omega<\omega$ in the resonators versus evolution time for the I1 system. The inset figure shows the mode temperature evolution in the membrane. The calculations are carried out at $100 \mathrm{~K}$.

ing the resonant nature of synchronization, a frequency decomposition is proposed. We hence define the mode temperature $^{44}$ of the resonators for different frequency intervals,

$$
\tilde{T}(t)=\frac{1}{k_{B} m n_{a} n_{\omega}} \sum_{i}\left|\int_{\omega_{1}}^{\omega_{2}} v_{i, \alpha}(t) e^{i \omega t} d \omega\right|^{2}
$$

where $m$ corresponds to the mass of a silicon atom and $n_{\omega}$ is the number of terms in the discrete summation. To study the mode dependent information, three integration intervals limited by $\omega_{1}$ and $\omega_{2}$ are taken into account: I) $\omega_{1}=0 ; \omega_{2}=\omega_{s}-\Delta \omega$, II) $\omega_{1}=\omega_{s}-\Delta \omega ; \omega_{2}=\omega_{s}+\Delta \omega$, and III) $\omega_{1}=\omega_{s}+\Delta \omega ; \omega_{2}=\infty . \Delta \omega$ is the frequency broadening of the peak at $\omega_{s}$. For the I1 system, $\omega_{s}$ and $\Delta \omega$ are respectively fixed at $0.152 \mathrm{THz}$ and $0.014 \mathrm{THz}$.

The mode temperature values are the sums over phonon modes with frequencies included in each interval. The mode temperatures are reported in Fig. 5. During self-synchronization before $300 \mathrm{ps}$, the potential energy is mainly converted into thermal energy around the frequency $\omega_{s}$. In other words, the increased kinetic energy between resonators is mostly reflected in the increase of the mode energy at $\omega_{s}$, which should originates from the amplification of phonon population for this mode. This indicates that synchronization results from the activation of thermal vibrations with frequencies near the pillar resonance. Strikingly, the amplitude of the thermal energy at frequency $\omega_{s}$ appears to be proportional to the phase synchronization degree.

Furthermore, Fig. 5 shows that in the vicinity of 300 ps, the thermal energy at frequency $\omega_{s}$ transfers to other modes inside the pillars. We presume that the energy transfer from the resonance frequency to other modes is mediated via phonon-phonon scattering, for instance via annihilation processes such as $\omega_{s} \rightarrow \omega^{\prime}+\omega^{\prime \prime 45,46}$. As scattering events accumulate, pillars follow a desynchronization process in the $300-500$ ps interval as reported in Fig. 5. Accordingly, phonon-phonon scattering resistively contributes to synchronization and is at the source of the entropy production $\Pi$ in Eq. (1). This dissipation is analogous to the energy cost of synchronization in biological systems ${ }^{8}$. It should be noted that the resonant vibrations in pillars are highly localized. In consequence, the energy transfer to the membrane occurs at a low rate as shown in the inset of Fig. 5.

Eq. (1) implies that self-synchronization demands a continuous external driving to offset the dissipative role of phonon-phonon scattering. Fig. 5 corroborates that the corresponding dissipated energy is converted into the thermal energy of other modes. The consequent desynchronization process is expected to increase again the distance and the potential energy between pillars, which leads to another potential energy minimization phase similar to the initial one. The previously described sequence is forming a cycle and finally an energy balance is established between the phonon-phonon scattering resulted dissipative energy and the potential energy, respectively corresponding to the contributions of $\Pi$ and $\Phi$ in Eq. (1). A macroscopic equilibrium state can be found after $\sim 500 \mathrm{ps}$, in which the phase synchronization degree (Fig. 3) and the mode energy $(\sim \tilde{T})$ at $\omega_{s}$ are converged. The schematic of Fig. 6 illustrates the above mentioned process involving the interplay between kinetic $\left(E_{k}\right)$ and potential $\left(E_{p}\right)$ energies.

We can further understand the above balance from the Kuramoto model. Henrich et al. ${ }^{12,40}$ proposed a Kuramoto-type model to consider the damping/energy dissipation effect. The simplified form reads

$$
\delta \dot{\varphi}=-\delta \Omega-[C \cos (\delta \varphi)+K \sin (2 \delta \varphi)] .
$$

The coupling constants between two resonators can be given by $C=\left(\xi_{12}-\xi_{21}\right) / 2, K=\left(\xi_{12}+\xi_{21}\right)^{2} / 8 \gamma . \xi_{i j}=$ $\phi_{i j} \bar{A}_{j} / M_{i} \Omega_{i} \bar{A}_{i}$ where $\bar{A}_{i}$ is the averaged amplitude of the $i$-th resonator. The term $\phi_{i j} \bar{A}_{j}$ refers to the external coupling force between the mechanical resonators as discussed in ${ }^{12,40}$. Instead, the coupling force between pillars in our system should be defined as $\phi \Delta x$, where $\Delta x$ is the relative displacement between pillars and $\phi \equiv \phi_{i j} \equiv \phi_{j i}$ under the mean-field coupling. $\Delta x$ can be calculated from molecular dynamics simulations as a result of thermal fluctuations by averaging the atomic relative displacement between nearest neighbor pillars. $\gamma$ refers to the dissipation rate that is introduced by the phononphonon scattering in our system. By fitting the spectral energy density of the synchronization mode of the pillars with the Lorenzian function $\Psi /\left[\left(\omega-\omega_{s}\right)^{2}+\gamma^{2}\right]$, we can obtain $\gamma$, here $\Psi$ is the energy amplitude. As we introduced in Sec. II, the resonance mode, referring to the synchronization mode in synchronized system, domi- 


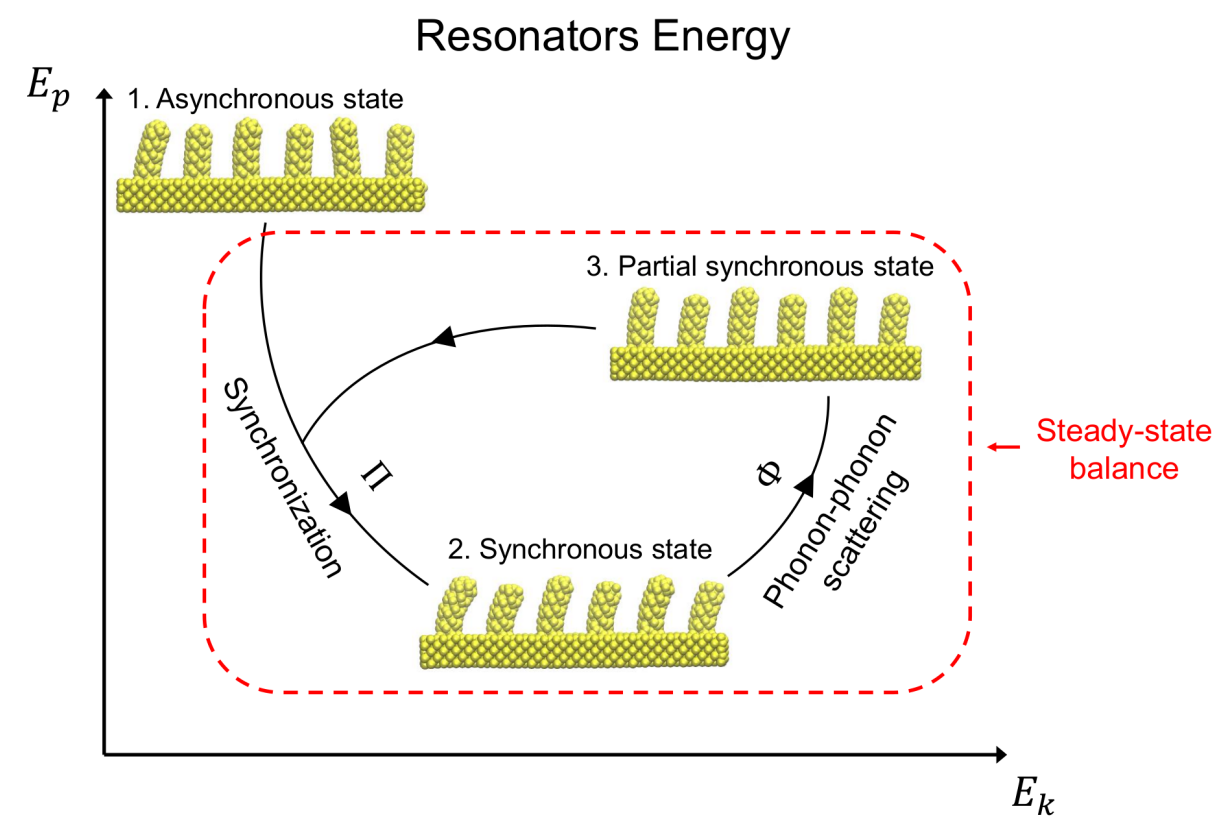

FIG. 6. Schematic of the equilibrium (constant entropy) state of self-synchronization in the resonators kinetic energy ( $E_{k}$ ) - potential energy $\left(E_{p}\right)$ plane. The process between state 1 and state 2 corresponds to the minimization of potential energy correlated to the reduction of the distance between pillars and to synchronization. The process between state 2 and state 3 is generated by the scattering of the resonant mode with other phonons. This dissipative phase increases kinetic energy but also potential energy via de-synchronization and the augmentation of inter-pillar distances. The amplification of potential energy feeds the synchronization process via its minimization (process between state 3 and state 2).

nates the vibrations in pillars, thus the fitting is only applied to the synchronization mode to manifest the damping in pillar resonators. The theory and calculation details about this fitting are well demonstrated $\mathrm{in}^{47}$. As discussed by Henrich et al. ${ }^{12,40}$, the constant $C$ in Eq. (11) can be neglected due to the weak coupling strength $\left(C \approx \phi \delta \Omega / 2 M \bar{\Omega}^{2} \ll \delta \Omega\right)$.

Now, if we consider the frequency synchronization for which $\delta \dot{\varphi}=0$, we find that the critical coupling constant $\left(\phi_{c}\right)$ is yielded as

$$
\phi_{c}=\frac{M \bar{\Omega} \bar{A} \sqrt{2 \delta \Omega \gamma}}{\Delta x} .
$$

Here, to simplify the formula, we consider the averaged mass $M$, averaged frequency $\bar{\Omega}$ and averaged amplitude $\bar{A}$ over different pillars. Compared to the $\phi_{c}$ appearing in the deterministic Kuramoto model described in Sec. III of this paper, Eq. (12) can simultaneously involve the effects of thermal fluctuation $(\Delta x)$ and energy dissipation $(\gamma)$. If $\phi_{c} \ll \phi_{\text {real }}$, where $\phi_{\text {real }}$ is the real harmonic coupling constant from the electrostatic field, the system can be easily synchronized. While, when $\phi_{c} \approx \phi_{\text {real }}$, the system can be fully synchronized in frequency but this condition might not be valid for phase synchronization. As we discussed in Sec. III, the phase synchronization is more difficult to achieve as compared to the frequency one.

The calculated relative displacement $\Delta x$ and dissipation rate $\gamma$ for the I1 system are shown Fig. $7(\mathrm{a})$ and

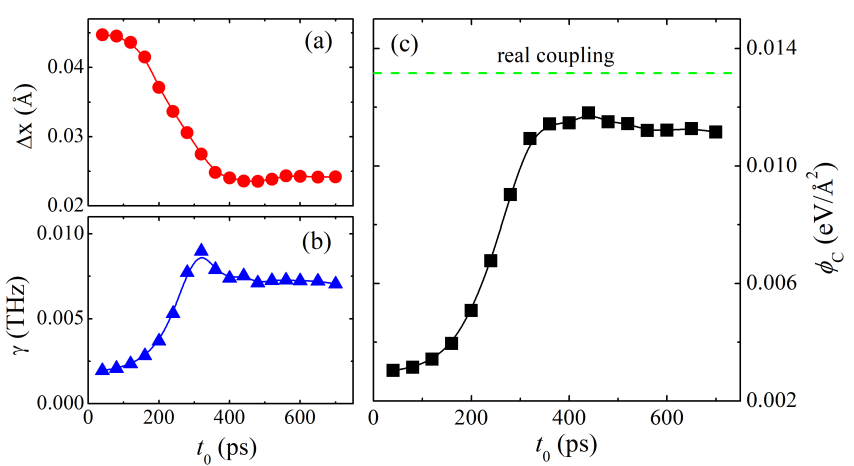

FIG. 7. The dynamics of the critical coupling constant. (a) The relative displacement $\Delta x$ between pillars and (b) the dissipation rate $\gamma$ at synchronization frequency versus evolution time. (c) The time-dependent critical coupling constant $\phi_{c}$ based on Eq. (12) with $\bar{A}=0.042 \AA$. The calculations are done for the I1 system at $100 \mathrm{~K}$. The dashed line in (c) is the real harmonic coupling constant $\phi_{\text {real }}$ between pillars.

(b). Those two quantities are time-dependent during the synchronization process, which further leads to a dynamical critical coupling constant in Fig. 7(c) according to Eq. (12). At the beginning, the random vibrations of the pillars result in a large $\Delta x$ and correspondingly in a small $\phi_{c}$. Thus the system can be rapidly synchronized due to the fulfilled condition $\phi_{c} \ll \phi_{\text {real }}$ (See Fig. $7(\mathrm{c}))$. For the I1 system, $\phi_{\text {real }}=1.32 \times 10^{-2} \mathrm{eV} / \AA^{2}$ that 
is larger than $\phi_{c}$. This result agrees well with the rapid synchronization of frequency and phase before $300 \mathrm{ps}$ in Fig. 2(a) and 3(a).

On the other hand, during this synchronizing process, $\Delta x$ is decreasing and the potential energy is converted into kinetic energy, which eventually enhances the dissipation $(\gamma)$ (See Fig. 7(b)). As a result, those two effects make $\phi_{c}$ increase and converge to the real coupling constant $\phi_{\text {real }}$. Finally, this convergence is observed after 500 ps in Fig. 7(c). In some degree, this trend results in the phase synchronization process in Fig. 3(a). In addition, the convergence trend indicates that the two independent effects related to $\Delta x$ and $\gamma$ in Eq. (12), accordingly thermal fluctuation and thermal dissipation, and lead to a balance. In the end, the condition $\phi_{c} \approx \phi_{\text {real }}$ means that the system can still be fully synchronized in frequency (See Fig. 2(a)), while the phase synchronization can only be partial in the equilibrium-state (See Fig. $3(\mathrm{a})$ ). Note that although the coupling and damping are correlated in realistic systems, the validation of their separate description in the Kuramoto model has been verified in the above discussion and also other phonon systems $^{12,40}$.

Moreover, the doping ratio also allows us to control the potential energy between pillars, by tuning the coupling constant $k$ appearing in the potential energy expression $\frac{1}{2} k \Delta x^{2}$, where $\Delta x$ is the relative displacement between pillars. Obviously, doping can improve the balance between phonon-phonon scattering and potential energy by elevating the interaction and potential energy between pillars. As shown in Fig. 3(a), the final selfsynchronization degree in phase is significantly enhanced as doping ratio increase from $0.2 \%$ to $0.4 \%$.

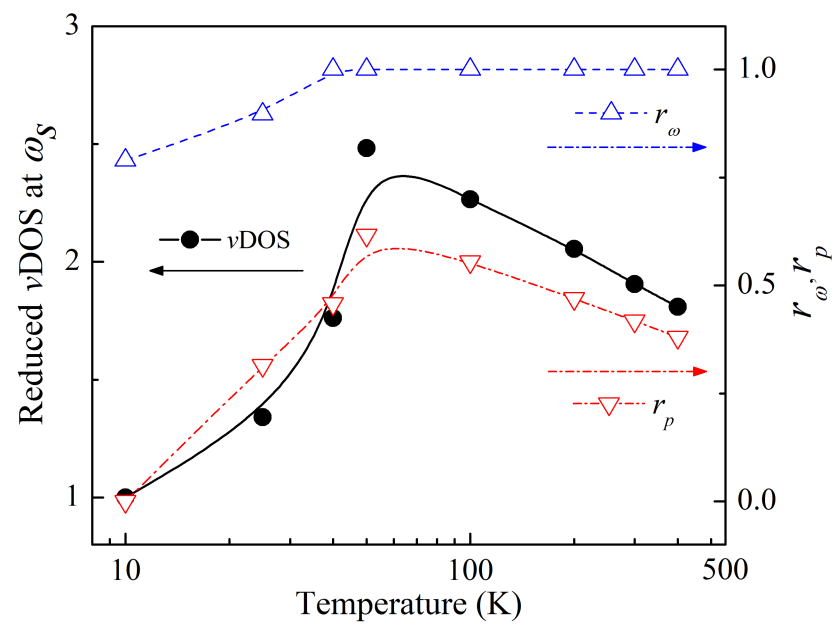

FIG. 8. Temperature effect on the synchronization of resonators. The $k_{B} T$ reduced $v D O S$ at synchronization frequency $\omega_{s}$ (left-axis) and synchronization degree in frequency $\left(r_{\omega}\right)$ and phase $\left(r_{p}\right)$ (right-axis) as a function of temperature for the I1 system. The temperature effect is simulated in stationary state in the NVE ensemble.

\section{Temperature effect on self-synchronization}

Temperature should have a significant effect on selfsynchronization, by affecting the stationary state. As temperature increases, a competition is raised, where temperature enhances the phonon-phonon scattering for de-synchronization but also elevates potential energy by increasing the amplitude of the displacements $\Delta x$. Accordingly, at low temperatures with weak phonon-phonon scattering, the increase of temperature should promote the synchronization by increasing the potential energy via thermal fluctuations (See Fig. 8), as manifested by the enhanced $r_{\omega}$ and $r_{p}$ in Fig. 6. However, as temperature continuously increases above $50 \mathrm{~K}$, phonon-phonon scattering becomes significant and suppresses phase synchronization degree $r_{p}$. In addition, the $k_{B} T$ reduced $v D O S$ at synchronization frequency $\omega_{s}$ is used to study the dynamics of synchronization, which exhibits the same trend than phase synchronization $r_{p}$. The Fig. 8 also evidences that a high synchronization degree in frequency is clearly much easier to achieve and more stable with temperature than a high $r_{p}$.

\section{The generation of coherent thermal phonons}

Even in the partial synchronization state, resonators should be collectively locked in frequency and phase, in some degree, exhibiting a coherent state. As thermal excitations become coherent in phase in a monochromatic mode, they behave as coherent (i.e. wavelike) thermal phonons. Recent works found that coherent thermal phonons play an important role in the thermal transport of phononic crystals and complex crystals $^{22-28}$. The effect of self-synchronization on the generation of coherent thermal phonons is studied in this section. Considering that phonons with different wavevectors and frequencies are thermally activated in MD simulations, the normal mode decomposition is applied to extract the dynamic modal information $(F(t))$ for a single mode. Here, the resonance mode at the Gamma point is studied (See the details of normal mode decomposition calculation in Appendix E). Previous studies ${ }^{48-50}$ demonstrated that the temporal coherence of thermal phonons can be analyzed by a wavelet transform approach as follows

$$
\Lambda\left(\omega_{s}, t_{0}, \tau_{s}^{c}\right)=\int \psi_{\omega_{s}, t_{0}, \tau_{s}^{c}}(t) F(t) d t
$$

where $\psi_{\omega_{s}, t_{0}, \tau_{s}^{c}}(t)$ is the wavelet basis. The temporal coherence of thermal phonons can be defined in the basis

$$
\psi_{\omega_{s}, t_{0}, \Delta_{s}}(t)=\pi^{-\frac{1}{4}} \Delta_{s}^{-\frac{1}{2}} e^{\left[i \omega_{s}\left(t-t_{0}\right)\right]} e^{\left[-\frac{1}{2}\left(\frac{t-t_{0}}{\Delta_{s}}\right)^{2}\right]},
$$

where $\omega_{s}$ is the angular frequency of the resonance/synchronization mode, and $\Delta_{s}$ defines the 

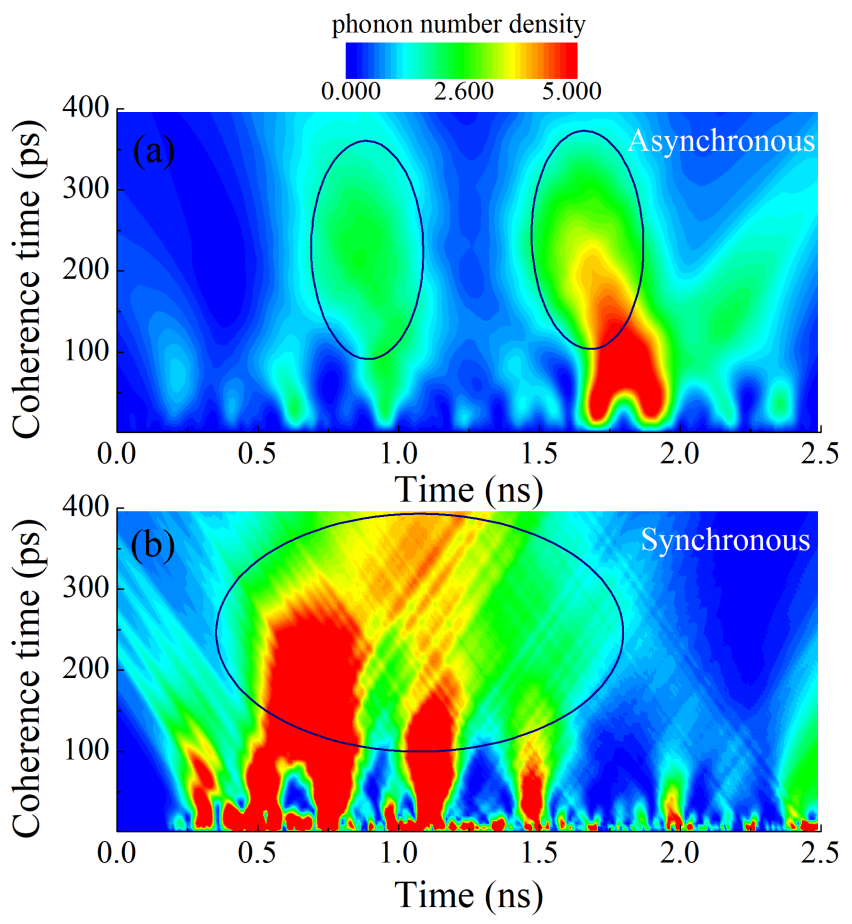

FIG. 9. Generation of coherent thermal phonons. Evolution time and coherence time dependent phonon number density for the thermal phonons in the (a) asynchronous state and (b) synchronous state. The calculation for the asynchronous state is performed in the $\delta \Omega_{I}$ system without the long-range electrostatic coupling (intrinsically asynchronous). In contrast, the calculation for the synchronous state is performed in the I1 system, as the system has reached a stable synchronous state after $600 \mathrm{ps}$. We have set this time point as the beginning of the evolution time in (b). The circles highlighting clouds indicate the zones where coherent thermal phonons emerge. The calculations are carried out at $100 \mathrm{~K}$.

wavepacket duration. $t$ corresponds to the time variable, and $t_{0}$ to the position of highest amplitude in the wavepacket and also corresponds to the time evolution in the wavelet space. Inside the wavepacket, planewaves are in phase, the $\Delta_{s}$ term in Eq. (14) is thus a measure of the temporal coherence of thermal phonons. Here, we define the wavepacket full-width at half-maximum (FWHM) as the coherence time $\tau_{s}^{c}=2 \sqrt{2 \ln 2} \Delta_{s}$. At the frequency $\omega_{s}$, the time dependent phonon number of a given coherence time, here called phonon number density, $N\left(t_{0}, \tau_{s}^{c}\right)$ can be calculated as $N\left(t_{0}, \tau_{s}^{c}\right)=\frac{1}{2} m\left|\Lambda\left(\omega_{s}, t_{0}, \tau_{s}^{c}\right)\right|^{2} / \hbar \omega_{s}$.

Figure 9 shows the calculated evolution time and coherence time dependent phonon number density $N\left(t_{0}, \tau_{s}^{c}\right)$ of the I1 system. Compared to the phonon number density of a synchronous state, we find that thermal phonons in the asynchronous system are mostly distributed in the short coherence time regions. In addition, the clouds of phonon number density show rapid phonon creation and annihilation evolutions, i.e. a short lifetime. After synchronizing, thermal phonons exhibit a different coherence behavior in Fig. 9(b). The coherence time-dependent mode occupation number $N\left(t_{0}, \tau_{s}^{c}\right)$ is extended both along evolution time and coherence time. Obviously, the emergence of phonon 'clouds' indicates the generation of new thermal phonons with long temporal coherence through the self-synchronization process. Moreover, thermal phonons with a long coherence time also exhibit a longer lifetime.

Note that in the synchronization state, i.e., in a strong nonlinear system, the application of Green-Kubo formalism becomes problematic, for instance in the calculation of the thermal conductivity based on the Green-Kubo approach. Nevertheless, the Green-Kubo approach is viable when the electrostatic force is much reduced or cancelled, bringing the system back to a linear state ${ }^{38}$.

\section{CONCLUSIONS}

By performing MD simulations, we have demonstrated self-synchronization of a collection of pillar resonators due to the activation by thermal fluctuations. We find that pillars are spontaneously self-synchronized in both frequency and phase (in partially). Phonon dynamics is analyzed based on the evolution of frequency and phase synchronization. In addition, the results show, in comparison to synchronization in frequency, that the phase synchronization is harder to be achieved and to stabilize, which agrees well with the predictions of the $\mathrm{Ku}$ ramoto model. The synchronization degree in phase is sensitive to the intrinsic frequency difference and the coupling strength between oscillators. Small frequency differences and strong coupling would enhance the synchronization. More interestingly, we find that there is a competing balance between energy dissipation resulting from phonon-phonon scattering and potential energy between resonators to maintain the stationary state of partial phase synchronization. Phonon-phonon scattering destroys the synchronized state but increases potential energy, while potential energy reversely feeds synchronization. This mechanism is further verified through the study of coupling strength and temperature effect.

In bulk materials, thermal phonons can also be coupled through covalent bonding or long-range interactions. We hence expect self-synchronization to also exist in phonon baths. In some extent, sufficient phonon-phonon scattering would suppress the synchronization degree and also its stability. On the other hand, coherent thermal phonons play an important role in thermal transport. Therefore, self-synchronization could be a promising approach for tuning coherent thermal phonons and also thermal conductivity. Eventually, we claim that the self-synchronization of thermal phonons is a new viewpoint for the generation of thermal phonons with long coherence times and lifetimes. Our findings are likely to advance the understanding of phonons and nano-objects dynamics from an unexpected perspective. 


\section{SUPPLEMENTARY MATERIAL}

The video in Supplementary Material records the timedependent atomic vibrations from the MD simulation. The video shows that at the beginning the vibrations of pillars are highly out-of phase, i.e. incoherent, with large relative displacement between pillars. During the synchronization process, i.e. after switched to NVE ensemble, the relative displacement is reduced and pillars are vibrating in phase, indicating the appearance of synchronization state.

\section{ACKNOWLEDGMENTS}

This project is supported in part by the grants from the National Natural Science Foundation of China (Grant Nos. 12075168 and 11890703), and Science and Technology Commission of Shanghai Municipality (Grant Nos. 19ZR1478600 and 18JC1410900). This work is partially supported by CREST JST (No. JPMJCR19Q3). Z. Z. gratefully acknowledge financial support from China Scholarship Council.

\section{APPENDIX A. PHONON DISPERSION}

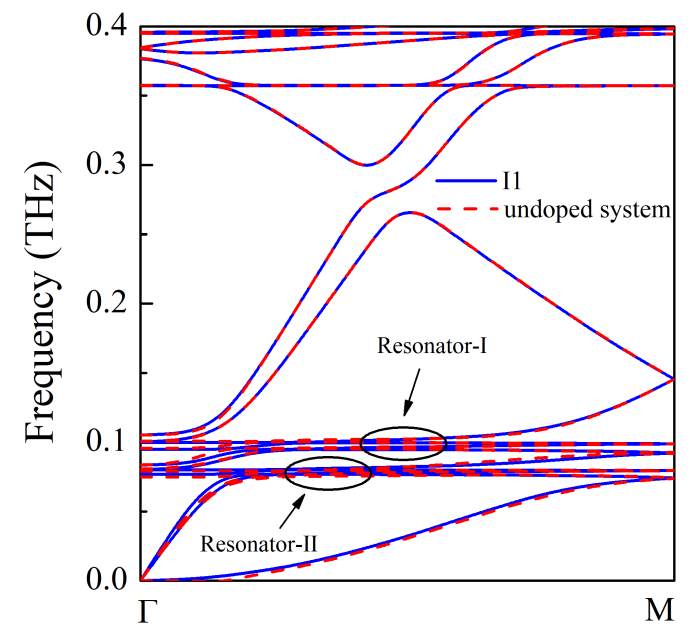

FIG. 10. Phonon dispersion from the harmonic approximation $(0 \mathrm{~K})$ of the I1 and undoped systems.

The phonon dispersion from harmonic approximation in Figure 10 shows that the electrostatic interaction in the doped system has negligible effects on the intrinsic frequencies of the resonators. Thus, the change of resonance frequency in the synchronization state should be related to the thermal fluctuations.

\section{APPENDIX B. THERMOSTAT EFFECT}

Figure 11 shows that the thermostat has negligible effects on the both frequency and phase synchronization process, and also the final synchronization degrees. This indicates that under the NVT ensemble the thermal vibrations of the system are fully desynchronized/incoherent, and the synchronization is spontaneously starting in the NVE ensemble (after 0 ps in Figure 11).
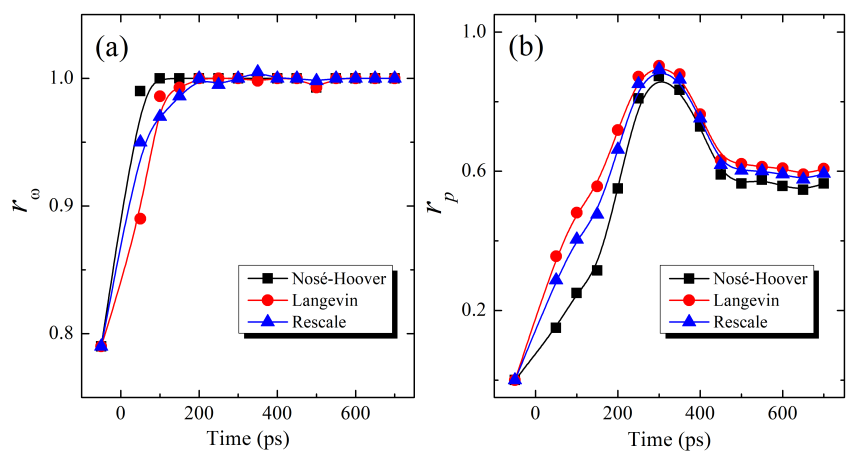

FIG. 11. (a) Frequency synchronization degree and (b) phase synchronization degree versus evolution time for I1 system at $100 \mathrm{~K}$ under different initial thermostats.

\section{APPENDIX C. VIBRATIONAL DENSITY OF STATES}

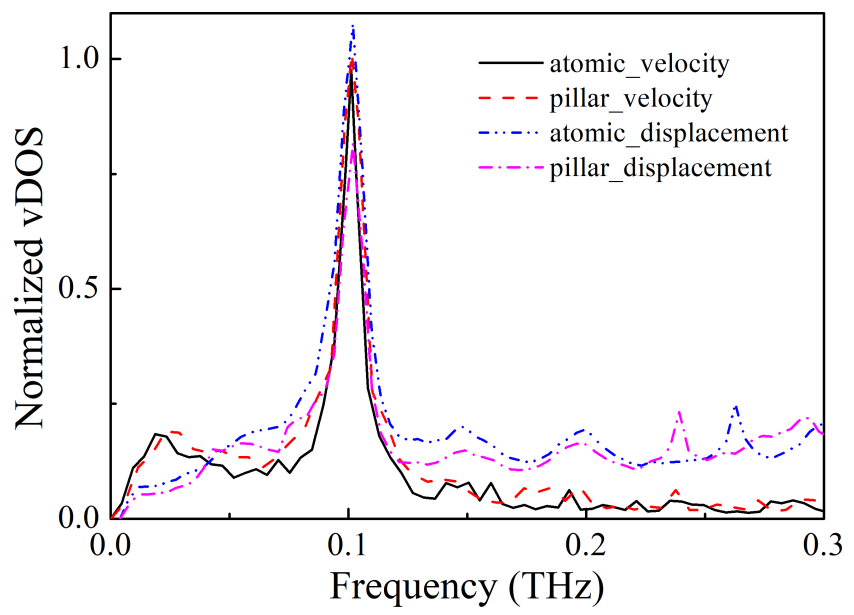

FIG. 12. The calculations of vibrational density of states from different quantities. The simulations are perform for the system with pillar height $1.09 \mathrm{~nm}$ at $100 \mathrm{~K}$.

Figure 12 indicates that the calculated vDOS respectively from atomic velocity or atomic displacement show the same vibrational information at the resonance frequency. In addition, due to the dominance of resonant vibrations in pillars, as averaged over different atoms in 
each pillar, the vDOS can also provide the same resonance frequency from velocity and displacement.

\section{APPENDIX D. TIMESCALE AND MEMBRANE SIZE EFFECT}

Figure 13 shows that the energy conversion is rapidly converged. The high stability of the energy conversion is consistent with the stable state of synchronization in Figs. 2 and 3. Note that the energy change of the membrane is constrained by the limited size of connection, i.e. the neck part, between pillars and membrane.

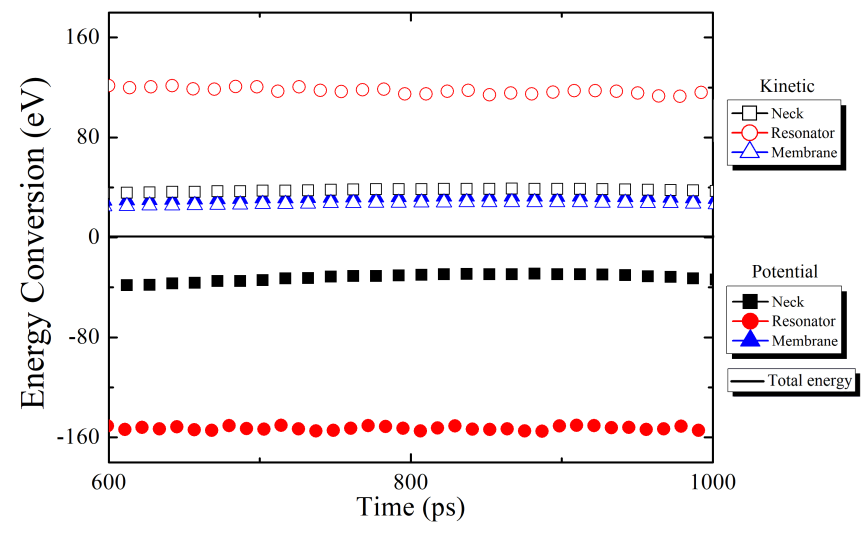

FIG. 13. Longer timescale simulation for the results in Fig. 4.

The membrane size effect on energy conversion is further studied in Fig. 14. Indeed, the energy conversion inside pillars is suppressed by increasing the membrane size, which could further reduce the synchronization between pillars. However, the energy exchange between pillars and membrane is still limited, and weak size dependence is displayed, due to the fixed size of the neck part and the intensively confined resonant vibration of pillars.

\section{APPENDIX E. NORMAL MODE DECOMPOSITION}

In the realistic system or our MD simulations, the phonons are thermally activated and statistically distributed by involving different wavevectors and frequencies. As demonstrated before ${ }^{47}$, the normal mode decomposition method can be adopted to extract the modal information for single mode from the complex system. Here, the used phonon modal velocity yields

$$
F(t)=\frac{1}{a} \sum_{b, l}^{a}\left[\dot{\mathbf{u}}_{b l}(t) \cdot \mathbf{e}_{b}^{*}(\mathbf{k}, s) \times \exp \left(i \mathbf{k} \cdot \mathbf{R}_{0 l}\right)\right],
$$

where $\dot{\mathbf{u}}_{b l}(t)$ is the velocity of the $b$ th atom in the $l$ th unit cell at time $t, a$ is the number of cell, $\mathbf{e}^{*}(\mathbf{k}, s)$ the complex conjugate of the eigenvector of mode $\mathbf{k} s$, and $\mathbf{R}_{0 l}$

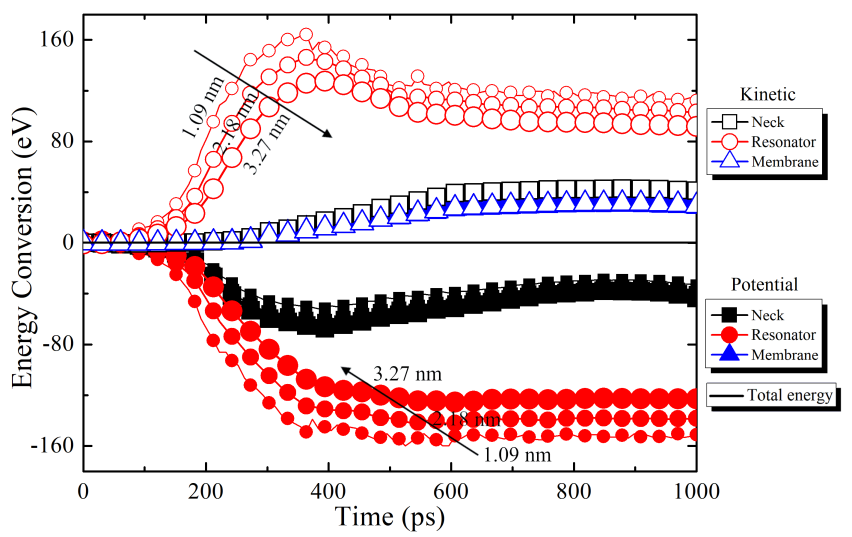

FIG. 14. Membrane size effect on energy conversion. The membrane thickness is fixed to $1.09 \mathrm{~nm}, 2.18 \mathrm{~nm}$ and 3.27 nm.

is the equilibrium position of the $l$ th unit cell. In this work, as we are focusing on the synchronization of resonators/pillars, the eigenvector $\mathbf{e}^{*}(\mathbf{k}, s)$ of the resonance frequency at Gamma is studied.

\section{AVAILABILITY OF DATA}

The data that support the findings of this study are available from the corresponding author upon reasonable request.

\section{REFERENCES}

${ }^{1}$ J. A. Acebrón, L. L. Bonilla, C. J. Pérez Vicente, F. Ritort, and R. Spigler, Rev. Mod. Phys. 77, 137 (2005).

${ }^{2}$ J. D. Touboul, C. Piette, L. Venance, and G. B. Ermentrout, Phys. Rev. X 10, 10.1103/PhysRevX.10.011073 (2020).

${ }^{3}$ L. L. Bonilla, J. M. Casado, and M. Morillo, J. Stat. Phys. 48, 571 (1987).

${ }^{4}$ M. F. Colombano, G. Arregui, N. E. Capuj, A. Pitanti, J. Maire, A. Griol, B. Garrido, A. Martinez, C. M. Sotomayor-Torres, and D. Navarro-Urrios, Phys. Rev. Lett. 123, 017402 (2019).

${ }^{5}$ J. Sheng, X. Wei, C. Yang, and H. Wu, Phys. Rev. Lett. 124, 053604 (2020).

${ }^{6}$ M. Zhang, G. S. Wiederhecker, S. Manipatruni, A. Barnard, P. McEuen, and M. Lipson, Phys. Rev. Lett. 109, 233906 (2012).

${ }^{7}$ G. Lan, P. Sartori, S. Neumann, V. Sourjik, and Y. Tu, Nat. Phys. 8, 422 (2012).

${ }^{8}$ D. Zhang, Y. Cao, Q. Ouyang, and Y. Tu, Nat. Phys. 16, 95 (2019).

${ }^{9}$ Y. Kuramoto, in International Symposium on Mathematical Problems in Theoretical Physics, edited by H. Araki (Springer Berlin Heidelberg) pp. 420-422.

${ }^{10}$ I. Hermoso de Mendoza, L. A. Pachon, J. Gomez-Gardenes, and D. Zueco, Phys. Rev. E 90, 052904 (2014).

${ }^{11}$ Y. Kuramoto and I. Nishikawa, J. Stat. Phys. 49, 569 (1987).

${ }^{12}$ G. Heinrich, M. Ludwig, J. Qian, B. Kubala, and F. Marquardt, Phys. Rev. Lett. 107, 043603 (2011).

${ }^{13}$ M. Zhang, S. Shah, J. Cardenas, and M. Lipson, Phys. Rev. Lett. 115, 163902 (2015).

${ }^{14}$ M. Ludwig and F. Marquardt, Phys. Rev. Lett. 111, 073603 (2013). 
${ }^{15}$ G. M. Süel, J. Garcia-Ojalvo, L. M. Liberman, and M. B. Elowitz, Nature 440, 545 (2006).

${ }^{16}$ T. Tome and M. J. de Oliveira, Phys. Rev. E 82, 021120 (2010).

${ }^{17}$ I. Prigogine and P. V. Rysselberghe, Journal of The Electrochemical Society 110, 97C (1963).

${ }^{18}$ T. Tome and M. J. de Oliveira, Phys. Rev. Lett. 108, 020601 (2012).

${ }^{19}$ I. I. Blekhman, A. L. Fradkov, O. P. Tomchina, and D. E. Bogdanov, Math. Comput. Simul. 58, 367 (2002).

${ }^{20}$ Y. Yamaguchi, K. Kometani, and H. Shimizu, J. Stat. Phys. 26, 719 (1981).

${ }^{21}$ Y. Kuramoto, Chemical oscillations, waves, and turbulence (Courier Corporation, 2003)

${ }^{22}$ S. Lee, K. Esfarjani, T. Luo, J. Zhou, Z. Tian, and G. Chen, Nat. Commun. 5, 3525 (2014).

${ }^{23}$ Z. Zhang, S. Hu, T. Nakayama, J. Chen, and B. Li, Carbon 139, 289 (2018).

${ }^{24}$ M. N. Luckyanova, J. Garg, K. Esfarjani, A. Jandl, M. T. Bulsara, A. J. Schmidt, A. J. Minnich, S. Chen, M. S. Dresselhaus, and Z. Ren, Science 338, 936 (2012).

${ }^{25} \mathrm{~S}$. Hu, Z. Zhang, P. Jiang, J. Chen, S. Volz, M. Nomura, and B. Li, J. Phys. Chem. Lett. 9, 3959 (2018).

${ }^{26}$ J. Maire, R. Anufriev, R. Yanagisawa, A. Ramiere, S. Volz, and M. Nomura, Sci. Adv. 3, e1700027 (2017).

${ }^{27}$ Y. Wang, H. Huang, and X. Ruan, Phys. Rev. B 90, 165406 (2014).

${ }^{28}$ Z. Zhang, Y. Ouyang, Y. Cheng, J. Chen, N. Li, and G. Zhang, Phys. Rep. 860, 1 (2020).

${ }^{29}$ H. Honarvar and M. I. Hussein, Phys. Rev. B 97, 195413 (2018).

${ }^{30}$ M. I. Hussein, C. Tsai, and H. Honarvar, Adv. Funct. Mater. 10.1002/adfm.201906718 (2019).

${ }^{31}$ S. Xiong, K. Sääskilahti, Y. A. Kosevich, H. Han, D. Donadio, and S. Volz, Phys. Rev. Lett. 117, 025503 (2016).
32 Z. Wei, J. Yang, K. Bi, and Y. Chen, J. Appl. Phys. 118, 155103 (2015).

${ }^{33}$ C. Li, M. Bescond, and M. Lannoo, Phys. Rev. B 80, 195318 (2009).

34 J. Tersoff, Phys. Rev. B 39, 5566 (1989).

${ }^{35}$ R. W. Hockney and J. W. Eastwood, Computer simulation using particles (crc Press, 1988).

${ }^{36}$ S. Plimpton, J. Comput. Phys. 117, 1 (1995).

${ }^{37}$ W. G. Hoover, Phys. Rev. A 31, 1695 (1985).

${ }^{38}$ B. L. Davis and M. I. Hussein, Phys. Rev. Lett. 112, 055505 (2014).

${ }^{39}$ O. Hellman, I. A. Abrikosov, and S. I. Simak, Phys. Rev. B 84 , 180301(R) (2011).

${ }^{40} \mathrm{G}$. Heinrich, Nanomechanics interacting with light: Dynamics of coupled multimode optomechanical systems Nanomechanik im Wechselspiel mit Licht: Dynamik gekoppelter optomechanischer Multimodensysteme, Thesis (2011).

${ }^{41}$ I. Andricioaei and M. Karplus, J. Chem. Phys. 115, 6289 (2001).

${ }^{42}$ M. Karplus and J. N. Kushick, Macromolecules 14, 325 (1981).

${ }^{43}$ J. W. Ponder and F. M. Richards, J. Comput. Chem. 8, 1016 (1987).

${ }^{44}$ T. Feng, W. Yao, Z. Wang, J. Shi, C. Li, B. Cao, and X. Ruan, Phys. Rev. B 95, 195202 (2017).

${ }^{45}$ M. Omini and A. Sparavigna, Phys. B: Condens. Matter 212, 101 (1995).

${ }^{46}$ K. Sääskilahti, J. Oksanen, J. Tulkki, and S. Volz, Phys. Rev. B 90, 134312 (2014).

${ }^{47}$ J. M. Larkin, J. E. Turney, A. D. Massicotte, C. H. Amon, and A. J. H. McGaughey, J. Comput. Theor. Nanosci. 11, 249 (2014). ${ }^{48}$ C. H. Baker, D. A. Jordan, and P. M. Norris, Phys. Rev. B 86, 104306 (2012).

49 J. Shiomi and S. Maruyama, Phys. Rev. B 73, 205420 (2006).

${ }^{50}$ Z. Zhang, Y. Guo, M. Bescond, J. Chen, M. Nomura, and S. Volz, Phys. Rev. B 103, 184307 (2021). 